\title{
Semidefinite Relaxations of the Quadratic Assignment Problem in a Lagrangian Framework
}

\author{
Frédéric Roupin \\ CEDRIC-CNAM, 292 rue Saint-Martin 75141 Paris cedex 03, France. \\ roupin@cnam.fr
}

\begin{abstract}
In this paper, we consider partial Lagrangian relaxations of continuous quadratic formulations of the Quadratic Assignment Problem (QAP) where the assignment constraints are not relaxed. These relaxations are a theoretical limit for semidefinite relaxations of the QAP using any linearized quadratic equalities made from the assignment constraints. Using this framework, we survey and compare standard semidefinite relaxations of this classical NP-hard problem. In particular, this approach is a simple way to prove that some well-known semidefinite relaxations for the QAP are equivalent. Nevertheless, these relaxations have a different numerical behavior and practical usefulness depending on the semidefinite programming solver. We discuss such issues by reporting some computational experiments.
\end{abstract}

Keywords: Quadratic Assignment Problem, Semidefinite Programming, Lagrangian relaxation.

\section{Introduction}

The Lagrangian approach provides a unified framework which helps to design and compare linear and non-linear relaxations for many combinatorial problems (Geoffrion 1974, Poljak, Rendl and Wolkowicz 1995, Guignard 2003, Lemaréchal 2003). For a given problem, there are usually several possible Lagrangian relaxations and refinement techniques. A recent example is (Litvinchev 2007) where the author obtains an improved Lagrangian bound by introducing an auxiliary problem involving complementary slackness conditions of linear programs. Besides, although some of these relaxations are not computationally tractable, others have a practical value since they can be explicitly stated as familiar mathematical programs (and thus one can solve them efficiently with standard solvers). For instance, a particular semi-Lagrangian relaxation is presented in (Beltran, Tadonki and Vial 2006). This relaxation generates an optimal integer solution for a $0-1$ linear programming formulation of the $p$-median problem which allows to solve large-scale instances. 
We are going to use such an approach to survey the semidefinite relaxations proposed for the Quadratic Assignment Problem (QAP), as well as their dominance relationships by comparing them with particular partial Lagrangian relaxations. The optimal values of these relaxations are actually a theoretical limit for the bounds one can obtain by using the standard semidefinite approach (Faye and Roupin 2007).

The paper is organized as follows. Section 2 is a short literature survey about the Quadratic Assignment Problem. In Section 3 we recall some results about the links between semidefinite, total and partial Lagrangian relaxations of general and 0-1 quadratic problems. Then, using these results, we survey and compare semidefinite relaxations for the QAP in Section 4. Finally, we discuss numerical issues by giving some experimental results in Section 5 .

\section{Literature survey}

The Quadratic Assignment Problem is a classical and very challenging NPhard combinatorial problem. It has brought considerable attention since many industrial and real life applications can be modeled as QAPs (F.Çela 1998): facilities location problems (Koopmans and Beckman 1957), scheduling problems, the traveling salesman problem, placement of interconnected electronic components such as the wiring of computer backboards (Steinberg 1961), dartboard design (Eiselt and Laporte 1991), typewriter keyboard design (Burkard and Offermann 1977). There exist several forms to state QAPs. Here, we shall use $(Q A P)$, the standard 0-1 quadratic formulation of the Quadratic Assignment Problem:

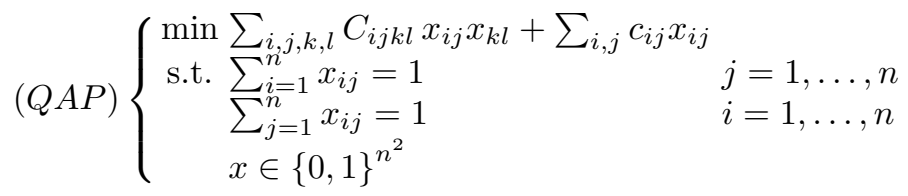

For instance, consider the case of a facilities location problem. The decision variable $x_{i j}$ equals 1 if and only if facility $i$ is assigned to location $j$, the "assignment constraints" being modeled by the linear constraints in $(Q A P)$. When facilities $i$ and $k$ are assigned to locations $j$ and $l$, respectively, $c_{i j}$ and $c_{k l}$ are the costs of this assignment, and $C_{i j k l}$ represents here the distance-flow product between locations $j$ and $l$.

In order to obtain lower bounds for the QAP, relaxations based on various mathematical programs have been studied : linear programming and polyhedral approaches (Adams and Johnson 1994, Kaibel 2000, Blanchard, Elloumi, Faye and Wicker 2003), convex quadratic bounds (Anstreicher and Brixius 2001, Anstreicher, Brixius, Goux and Linderoth 2002), and Reformulation-Linearization Techniques (Adams, Guignard, Hahn and Hightower 2007). It is beyond the scope of this paper to review all the exact or heuristic algorithms that have been 
proposed to solve the QAP. Therefore we refer the reader to (Anstreicher 2003) and (Loiola, de Abreu, Boaventura-Netto, Hahn and Querido 2007) for recent surveys.

We are going to focus on the Semidefinite Programming (SDP) approach which has been intensively studied during the last ten years because it provides tight lower bounds for the QAP (Karisch 1995, Zhao, Karish, Rendl and Wolkowicz 1998, Roupin 2004, Burer and Vandenbussche 2006, Povh and Rendl 2006, Rendl and Sotirov 2007, de Klerk and Sotirov 2007). We shall detail in section 4 the different models and SDP relaxations used in these papers. But as of now, let us point out that the design of these semidefinite relaxations has strongly depended on the SDP solvers available at the moment and even on memory limitations in some cases. In particular, the existence of strictly feasible points has played a crucial role. Moreover, authors have often used different stopping criterions in their numerical tests, and the bounds presented does not always correspond to the optimal values of the semidefinite relaxations. Besides, for some solvers (like SB (Helmberg 2004)), adding redundant constraints to the semidefinite programs (although it is theorically useless) can speed up the solving process. This means that paradoxically, solving an equivalent and larger semidefinite program can require less time (Faye and Roupin 2005). These experimental results can lead to the belief that some semidefinite relaxations are tighter than others, or (because of the chosen solver) are useless from a practical point of view. Hence, the main contribution of this paper is to obtain and compare all these semidefinite relaxations by using a standard Lagrangian framework, independently from the SDP solvers.

\section{Partial Lagrangian relaxations of 0-1 Quadratic problems}

In this section we recall some results about the links between partial Lagrangian and semidefinite relaxations of general and 0-1 quadratic programs. Consider $(Q)$, a 0-1 quadratic program which contains some linear equalities:

$$
(Q) \begin{cases}\min & x^{T} Q x+c^{T} x \\ \text { s.t. } & A x=b \\ & x^{T} B_{i} x+d_{i}^{T} x+e_{i}=(\text { or } \leq) 0 i \in I \\ & x \in\{0,1\}^{n}\end{cases}
$$

where $A$ is a $p \times n$ matrix, $I$ is a finite set of indices, $B_{i}$ are symmetric $n \times n$ matrices, $d_{i}$ are $n$-vectors, and $e_{i} \in \Re$. In order to obtain a continuous formulation of $(Q)$ we can replace the constraints $x \in\{0,1\}^{n}$ by $x_{k}^{2}=x_{k}$ for all $k \in\{1, \ldots, n\}$. In $0-1$ or general quadratic programming, a common approach to obtain better linear or semidefinite relaxations is adding valid quadratic equalities or inequalities to the initial problem then linearize them (Adams and Sherali 1986, Laurent 2003). From a Lagrangian point of view this can be seen 
as a reduction of the duality gap by dualizing these additional constraints. Consequently, we are interested here by the set of null quadratic functions over $\{x: A x=b\} \cap\{0,1\}^{n}$.

Recall that the set $\mathcal{J}$ of all quadratic constraints which are satisfied for $x$ such that $A x=b$ is characterized thanks to the following result proven in (Faye and Roupin 2007):

Theorem 1 A quadratic function is constant over $\mathcal{A}=\{x: A x=b\}$ if and only if it is equal to $F_{W, \alpha}(x)=x^{T}\left(A^{T} W^{T}+W A\right) x+\left(A^{T} \alpha-2 W b\right)^{T} x$, where $W$ is a $n \times p$-matrix, and $\alpha$ is a p-vector.

For vectors belonging to $\mathcal{A}$, one can verify that $F_{W, \alpha}(x)=\alpha^{T} b$. Hence, when applying a Lagrangian approach one can add to $(Q)$ any subset of $\mathcal{J}$ by choosing some matrices $W_{j}$ and vectors $\alpha_{j}$ for $j$ in $J$, a finite set of indices. In the general case, the set of null quadratic functions over $\{x: A x=b\} \cap\{0,1\}^{n}$ is obviously larger than $\mathcal{J}$. Nevertheless, for particular values of $A$ and $b$, this set is entirely described (see e.g. (Billionnet and Elloumi 1992)). Therefore, one can also consider to add to $(Q)$ a set $\mathcal{K}$ containing some of these valid quadratic constraints. This set $\mathcal{K}$ may also include any valid inequalities, for instance triangle inequalities or any others specific to $(Q)$. Hence, in the general case, an equivalent formulation of $(Q)$ that one can use in the first step of a Lagrangian approach is:

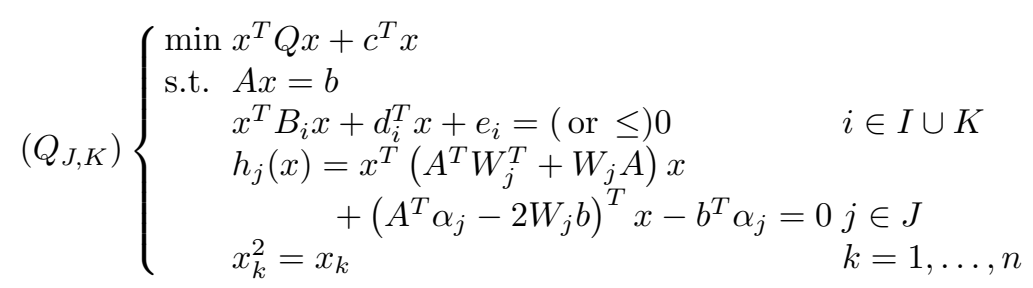

In the following, for better readability, the sign constraints over the Lagrange vectors will be assumed to hold when needed (and thus they will not be written). Denote by diag $(y)$ the diagonal matrix made from any real vector $y$ and define:

$$
\begin{gathered}
Q(\mu, \nu, \omega)=Q+\operatorname{diag}(\mu)+\sum_{i \in I \cup K} \omega_{i} B_{i}+\sum_{j \in J} \nu_{j}\left(A^{T} W_{j}^{T}+W_{j} A\right) \\
c(\mu, \nu, \omega, \lambda)=c-\mu+A^{T} \lambda+\sum_{i \in I \cup K} \omega_{i} d_{i}+\sum_{j \in J} \nu_{j}\left(A^{T} \alpha_{j}-2 W_{j} b\right) \\
e(\nu, \omega, \lambda)=\omega^{T} e-b^{T}\left(\sum_{j \in J} \nu_{j} \alpha_{j}+\lambda\right)
\end{gathered}
$$

Then the total Lagrangian relaxation of $\left(Q_{J, K}\right)$ is:

$$
\left(D T_{J, K}\right) \sup _{\mu, \nu, \omega, \lambda} \inf _{x \in \Re^{n}} x^{T} Q(\mu, \nu, \omega) x+c(\mu, \nu, \omega, \lambda)^{T} x+e(\nu, \omega, \lambda)
$$


The Lagrangian function being a simple unconstrained quadratic function, the semidefinite program $\left(S D P_{J, K}\right)$ is the dual of an explicit semidefinite formulation of $\left(D T_{J, K}\right)$ (see e.g. (Lemaréchal and Oustry 2001)):

$$
\left(S D P_{J, K}\right)\left\{\begin{array}{rlr}
\min & Q \bullet X+c^{T} x & \\
\text { s.t. } & B_{i} \bullet X+d_{i}^{T} x+e_{i}=(\text { or } \leq) 0 \quad \\
& A x=b \\
& H_{j}(X, x)=\left(A^{T} W_{j}^{T}+W_{j} A\right) \bullet X \\
& +\left(A^{T} \alpha_{j}-2 W_{j} b\right)^{T} x-b^{T} \alpha_{j}=0 j \in J \\
& X_{i i}=x_{i} \\
& {\left[\begin{array}{ll}
1 & x^{T} \\
x & X
\end{array}\right] \succeq 0}
\end{array}\right.
$$

where $A \bullet B=\sum_{i=1}^{n} \sum_{j=1}^{n} A_{i j} B_{i j}$ is the standard inner product of two $n \times n$ real symmetric matrices $A$ and $B$, and $A \succeq 0$ means that $A$ is positive semidefinite. Note that $\left(S D P_{J, K}\right)$ is a semidefinite relaxation of $\left(Q_{J, K}\right)$. Indeed, as shown in (Lemaréchal and Oustry 2001) in the general case, bidualizing $\left(Q_{J, K}\right)$ leads to a convexification of $(Q)$ into a semidefinite program.

Let us point out that in $\left(S D P_{J, K}\right)$, we can add to $H_{j}(X, x)=0$ any linear combination $\sum_{i=1}^{n} \beta_{i}\left(X_{i i}-x_{i}\right)$ (which is equal to zero). Thus, thanks to the continuous formulation of the 0-1 constraints, the general expression of redundant quadratic constraints which are used here is $x^{T}\left(A^{T} W^{T}+W A\right) x+$ $\left(A^{T} \alpha-2 W b\right)^{T} x-\alpha^{T} b+\sum_{i=1}^{n} \beta_{i}\left(x_{i}^{2}-x_{i}\right)=0$ where $W_{j} \in \Re^{n \times p}, \alpha \in \Re^{p}$ and $\beta \in \Re^{n}$.

Now, recall that $\mathcal{A}=\{x: A x=b\}$, and denote by $\left(D P_{J, K}\right)$, the partial Lagrangian relaxation of $\left(Q_{J, K}\right)$ where the constraints $A x=b$ are not relaxed. Then each constraint $h_{j}(x)=0$ is satisfied and does not appear in the associated partial Lagrangian function. Consequently, it is useless to add these constraints to $(Q)$ : for any sets $\mathcal{J}$ and $\mathcal{K}$ of additional constraints, we can only consider the partial Lagrangian relaxations:

$$
\begin{aligned}
& \left(D P_{K}\right) \\
& \sup _{\mu, \omega} \inf _{x \in \mathcal{A}} x^{T}\left(Q+\operatorname{diag}(\mu)+\sum_{i \in I \cup K} \omega_{i} B_{i}\right) x+\left(c-\mu+\sum_{i \in I \cup K} \omega_{i} d_{i}\right)^{T} x+\omega^{T} e
\end{aligned}
$$

It is straightforward to verify that given $\mathcal{K}$ and for any set of redundant quadratic constraints $\left\{h_{j}(x)=0: j \in J\right\} \subset \mathcal{J}$ the value of $\left(D T_{J, K}\right)$ is less or equal to the value of $\left(D P_{K}\right)$, since less constraints have been dualized.

In (Lemaréchal and Oustry 2001) the authors prove that if one uses $\mathcal{C}=$ $\left\{h(x)=x^{T} A^{T} A x-2 b^{T} A x+b^{T} b=0\right\} \subset \mathcal{J}$ then the optimal values of $\left(D T_{J, K}\right)$ and $\left(D P_{K}\right)$ are equal. This constraint, which can be seen as a penalty term in the Lagrangian function, is obtained by taking $\alpha=-b$ and $W=\frac{1}{2} A^{T}$ in 
the general formula of Theorem 1. But the existence of an optimal solution is not guaranteed, and thus the optimal value of the associated total Lagrangian relaxation may not be achieved by a feasible solution. An analogous result for general quadratic programming is given in (Faye and Roupin 2007): if $\left(\mu^{*}, \omega^{*}\right)$ is an optimal solution of $\left(D P_{K}\right)$ and if there exists a real vector $\nu$ such that $Q\left(\mu^{*}, \omega^{*}, \nu\right)$ is positive semidefinite then the values of $\left(D T_{J, K}\right)$ and $\left(D P_{K}\right)$ are equal. This hypothesis can be satisfied by choosing the set $\mathcal{P}$ (Faye and Roupin 2007):

$$
\mathcal{P}=\left\{h_{i j}(x)=\sum_{k=1}^{n} A_{j k} x_{k} x_{i}-b_{j} x_{i}=0 ; \forall i \in\{1, \ldots, n\}, \forall j \in\{1, \ldots, p\}\right\}
$$

These functions are obtained from the general formula of Theorem 1 by taking $\alpha_{i j}=0$ and $W_{i j}=\frac{1}{2} E_{i j}$, where $E_{i j}$ is a matrix with all zero entries except in the intersection row $i$, column $j$ where the entry equals 1 . In other words, these results allow to transform a constrained problem (a partial Lagrangian relaxation) into an unconstrained one (a total Lagrangian relaxation). Consequently, the two corresponding semidefinite relaxations $\left(S D P_{\mathcal{P}}\right)$ and $\left(S D P_{\mathcal{C}}\right)$ are equivalent. Note that for general quadratic programming, a direct proof without considering the dual programs is given in (Faye and Roupin 2007) when $A$ is a full rank matrix.

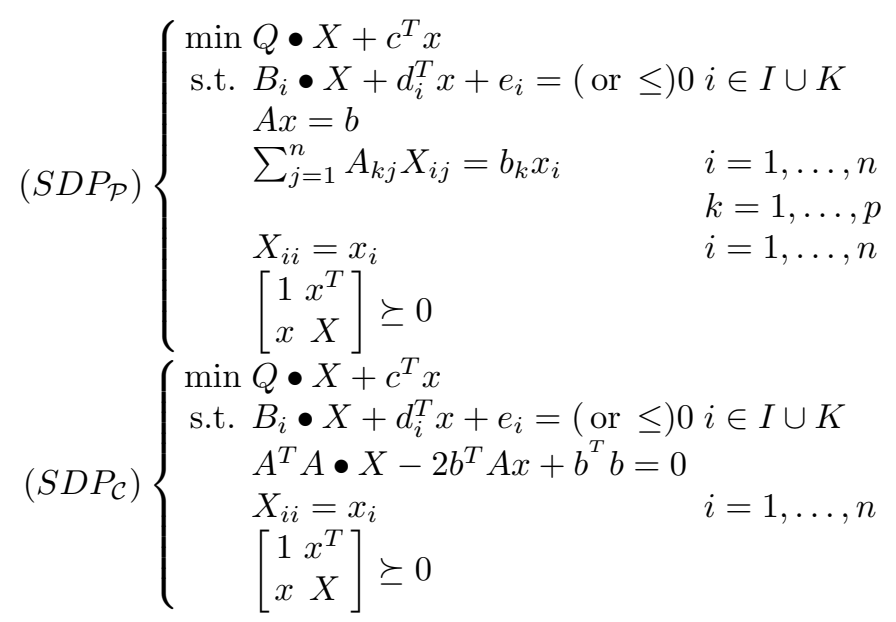

The constraint $A x=b$ has been removed from $\left(S D P_{\mathcal{C}}\right)$ because it is implied by $A^{T} A \bullet X-2 b^{T} A x+b^{T} b=0$ and $X-x x^{T} \succeq 0$. In summary, we can conclude that given a set $\mathcal{K}$, the optimal value of the best semidefinite relaxation that one can obtain by this approach is thus equal to the value of $\left(D P_{K}\right)$. Moreover, this value is achieved by the semidefinite programs $\left(S D P_{\mathcal{C}}\right)$ and $\left(S D P_{\mathcal{P}}\right)$. 


\section{Semidefinite relaxations of the QAP from a Lagrangian point of view}

Now, we are going to apply the results of the previous section to $(Q A P)$, the standard 0-1 quadratic formulation of the Quadratic Assignment Problem given in Section 2. The first step is to find the largest possible set of null quadratic functions over the feasible set of $(Q A P)$. This can be done by taking advantage of the particular values of $A$ and $b$.

Consider the Quadratic semi-assignment Problem (QSAP). This problem is a variant of QAP, and several classical combinatorial problems such as taskassignment or graph partitioning problems can be formulated as QSAP. It can be stated as:

$$
(Q S A P)\left\{\begin{array}{l}
\min \sum_{i, j, k, l} C_{i j k l} x_{i j} x_{k l}+\sum_{i, j} c_{i j} x_{i j} \\
\text { s.t. } \sum_{j=1}^{m} x_{i j}=1 \\
x \in\{0,1\}^{n \times m}
\end{array} \quad i=1, \ldots, n\right.
$$

In (Billionnet and Elloumi 1992), in order to to obtain the best reduction of this problem, the authors give the general expression of a null quadratic function $\Gamma(x)$ over the feasible set of $(Q S A P)$ :

$$
\Gamma(x)=\sum_{i=1}^{n} \lambda_{i} g_{i}(x)+\sum_{i=1}^{n} \sum_{k=1, k \neq i}^{n} \sum_{j=1}^{m} \delta_{i j k} x_{k j} g_{i}(x)+\sum_{i=1}^{n} \sum_{j=1}^{m-1} \sum_{l=j+1}^{m} \mu_{i j l} x_{i j} x_{i l}
$$

where $\lambda_{i}, \delta_{i j k}$, and $\mu_{i j l}$ are any real numbers and $g_{i}(x)=\sum_{j=1}^{m} x_{i j}-1$. This formula can be extended for the QAP straightforwardly by taking $m=n$ and by defining $g_{j}^{\prime}(x)=\sum_{i=1}^{n} x_{i j}-1$ for $j$ in $\{1, \ldots, n\}$. By this way, we get a similar function $\Delta(x)$ formed by three linear combinations of the functions $g_{j}^{\prime}(x)$, $x_{k i} g_{j}^{\prime}(x)$ and $x_{j i} x_{l i}$. It is easy to verify that $\Gamma(x)+\Delta(x)$ is a null quadratic function over the feasible of set $(Q A P)$. Conversely, we conjecture that any null quadratic function over the feasible set of $(Q A P)$ can be expressed in this form. In fact, we have obtained $n^{3}-n^{2}$ new elementary null quadratic functions over the feasible set of $(Q A P): f_{i j l}(x)=x_{i j} x_{i l}$ and $g_{i j l}(x)=x_{j i} x_{l i}$ for $i$ in $\{1, \ldots, n\}$, $j$ in $\{1, \ldots, n-1\}$ and $l>j$. Any linear combination of constraints made from these functions can be added to the set $\mathcal{K}$. The other functions are particular cases of $F_{W, \alpha}(x)+\sum_{i, j} \beta_{i}\left(x_{i j}^{2}-x_{i j}\right)$ (see Section 3 , and especially the set $\mathcal{P}$ ). Therefore, it is useless to add them to $\mathcal{K}$.

Now, following the approach recalled in Section 3, we can add any other valid inequalities to $\mathcal{K}$ then formulate the resulting problem as a continuous quadratic program by replacing the constraints $x \in\{0,1\}^{n^{2}}$ by $x_{i j}^{2}=x_{i j}$ for all $i$ and $j$ in $\{1, \ldots, n\}$ to obtain $\left(Q A P_{K}\right)$. 


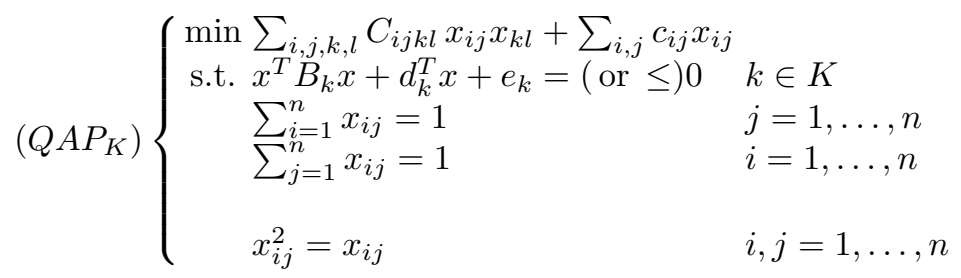

Here, $\mathcal{A}=\{x: A x=b\}$ is the set of $x \in \Re^{n^{2}}$ such that $\sum_{i=1}^{n} x_{i j}=1$ $\forall i \in\{1, \ldots, n\}$ and $\sum_{j=1}^{n} x_{i j}=1 \forall j \in\{1, \ldots, n\}$. Thus, the partial Lagrangian relaxation of $\left(Q A P_{K}\right)$ where the constraint $x \in \mathcal{A}$ is not relaxed is:

$$
\begin{aligned}
& (D P)_{Q A P}^{K} \\
& \sup _{\omega, \mu} \inf _{x \in \mathcal{A}} x^{T}\left(C+\operatorname{diag}(\mu)+\sum_{k \in K} \omega_{k} B_{k}\right) x+\left(\sum_{k \in K} \omega_{k} d_{k}+c-\mu\right)^{T} x+\omega^{T} e
\end{aligned}
$$

$\mu$ and $\omega$ are the Lagrangian vectors associated to the constraints $x_{i j}^{2}-x_{i j}=0$ and to the valid equalities and inequalities of $\mathcal{K}$, respectively. Thanks to the results of Section 3, we know that two (equivalent) semidefinite formulations of $(D P)_{Q A P}^{K}$ are $\left(Q A P_{\mathcal{P}}\right)^{K}$ and $\left(Q A P_{\mathcal{C}}\right)^{K}$.

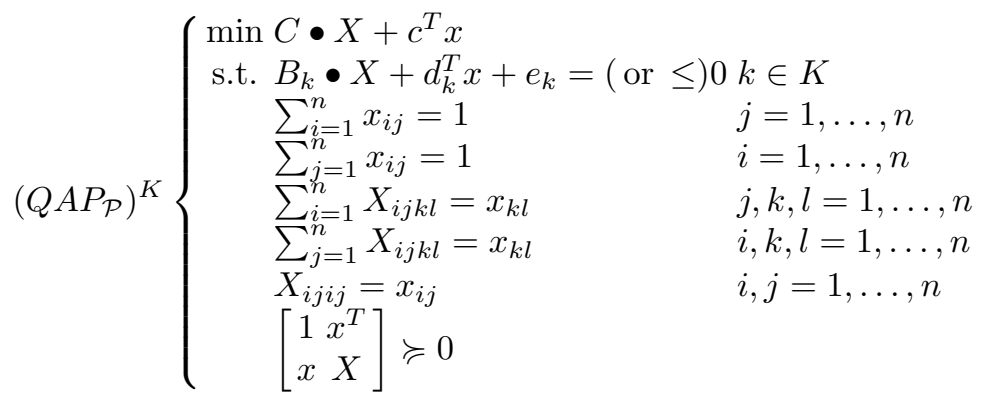

$$
\begin{aligned}
& \left(Q A P_{\mathcal{C}}\right)^{K}\left\{\begin{array}{rl}
\min & C \bullet X+c^{T} x \\
\text { s.t. } & B_{k} \bullet X+d_{k}^{T} x+e_{k}=(\text { or } \leq) 0 k \in K \\
& A^{T} A \bullet X-2 b^{T} A x+b^{T} b=0 \\
& X_{i j i j}=x_{i j} \\
& {\left[\begin{array}{ll}
1 & x^{T} \\
x & X
\end{array}\right] \succcurlyeq 0}
\end{array} \quad i, j=1, \ldots, n\right.
\end{aligned}
$$

Recall that we use the notations of section 3 for $\left(Q A P_{\mathcal{C}}\right)^{K}: A$ and $b$ (here the all ones $2 n$-vector) correspond to the assignment constraints. Now, by choosing different sets $\mathcal{K}$, we can survey the semidefinite relaxations of the QAP proposed in the literature.

The weakest relaxation is obtained of course by taking $\mathcal{K}=\varnothing .\left(Q A P_{\mathcal{P}}\right)^{\varnothing}$ (which is equivalent to $\left(Q A P_{\mathcal{C}}\right)^{\varnothing}$ ) is considered in (Karisch 1995) and denoted by 
$\left(Q A P_{\mathrm{R} 1}\right)$. This program has no strictly feasible points but, as recalled in (Burer and Vandenbussche 2006), the semidefinite dual of $\left(Q A P_{\mathcal{P}}\right)^{\varnothing}$ has an interiorpoint. Thus there is no duality gap and optimality is attained in $\left(Q A P_{\mathcal{P}}\right)^{\varnothing}$. Nevertheless, because of the algorithm used to solve this semidefinite program, an equivalent formulation is proposed in (Karisch 1995) to get into the relative interior of the feasible set:

$$
\left(Q A P_{R 1}\right)\left\{\begin{array}{l}
\min C \bullet X+c^{T} x \\
\text { s.t. } \hat{V} R \hat{V}^{T}=\left[\begin{array}{ll}
1 & x^{T} \\
x & X
\end{array}\right] \\
X_{i j i j}=x_{i j} \quad i, j=1, \ldots, n \\
R \succcurlyeq 0
\end{array}\right.
$$

where $R$ is a $\left((n-1)^{2}+1\right) \times\left((n-1)^{2}+1\right)$ real symmetric matrix, and $\hat{V}$ is a constant matrix (see (Karisch 1995) for details). Now, one can formulate $\left(Q A P_{R 1}\right)$ only by using the matrix $R$, and get an semidefinite program which admits strictly feasible points. In particular, it is proved in (Karisch 1995) (see lemma 4.3.6) that the feasible matrices $Z=\hat{V} R \hat{V}^{T}$ of $\left(Q A P_{R 1}\right)$ satisfy all the contrains of $\left(Q A P_{\mathcal{P}}\right)^{\varnothing}$. Hence, one can start from this formulation of $(D P)_{Q A P}^{\varnothing}$ or from $\left(Q A P_{\mathcal{P}}\right)^{\varnothing}$ to build stronger semidefinite relaxations for the QAP. In fact, the choice mainly depends on the method used to compute the bounds.

Now, take $\mathcal{K}=\mathcal{O}=\left\{\sum_{i=1}^{n} f_{i j l}(x)=\sum_{i=1}^{n} x_{i j} x_{i l}=0, \sum_{i=1}^{n} g_{i j l}(x)=\right.$ $\left.\sum_{i=1}^{n} x_{j i} x_{l i}=0: j<l ; j, l=1, \ldots, n\right\}$. These constraints are particular linear combinations of the null quadratic functions $f_{i j l}$ and $g_{i j l}$ over $\mathcal{A} \cap\{0,1\}^{n^{2}}$. In several papers, these constraints are called "orthogonality" contraints since they actually correspond to the inner products between two different lines (or columns) of $x$.

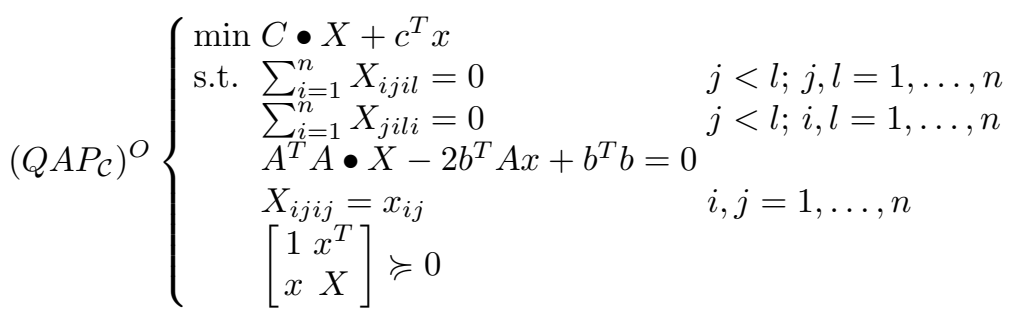

By this way, we obtain $\left(Q A P_{\mathcal{P}}\right)^{O}$ and $\left(Q A P_{\mathcal{C}}\right)^{O}$. As proposed in (Karisch $1995)$, one can add to $\left(Q A P_{R 1}\right)$ the same "orthogonality" contraints. This will lead to an equivalent relaxation, denoted by $\left(Q A P_{R 2}\right)$ in (Karisch 1995). Note that this latter semidefinite program and $\left(Q A P_{\mathcal{C}}\right)^{O}$ contain $O\left(n^{2}\right)$ linear contraints. Therefore, although the bounds obtained by using this set $\mathcal{K}$ are quite poor, they can be computed into a reasonable time (by an interior point method for the one presented in (Karisch 1995)). In a recent paper (Povh and Rendl 2006), a similar semidefinite relaxation is obtained from a copositive represen- 
tation of the QAP:

$$
\left(Q A P_{A W 1}\right)\left\{\begin{array}{rll}
\min & (C+\operatorname{diag}(c)) \bullet X \\
\text { s.t. } & \sum_{i=1}^{n} X_{i j i l}=0 & j<l ; j, l=1, \ldots, n \\
& \sum_{i=1}^{n} X_{j i l i}=0 & j<l ; j, l=1, \ldots, n \\
& \sum_{i=1}^{n} X_{i j i j}=1 & j=1, \ldots, n \\
& \sum_{j=1}^{n} X_{i j i j}=1 & i=1, \ldots, n \\
& \sum_{i, j, k, l} X_{i j k l}=n^{2} \\
& X \succcurlyeq 0
\end{array}\right.
$$

Recall that $\operatorname{diag}(c)$ is the diagonal matrix made from the vector $c$. Using the results of Section 3 and by noticing that $\sum_{i, j, k, l} X_{i j k l}=n^{2}$ is the linearization of the null quadratic function $\sum_{i, j, k, l} x_{i j} x_{k l}=n^{2}$ over $\mathcal{A}$, it is straightforward to verify that the optimal value of $\left(Q A P_{A W 1}\right)$ is less than or equal to the optimal value of $(D P)_{Q A P}^{\mathcal{O}}$ which is equivalent to $\left(Q A P_{\mathcal{C}}\right)^{\mathcal{O}}$ and $\left(Q A P_{R 2}\right)$.

For $\mathcal{K}=\mathcal{G}=\left\{x_{i j} x_{i l}=0, x_{j i} x_{l i}=0: j \neq l ; i, j, l=1, \ldots, n\right\},\left(Q A P_{\mathcal{P}}\right)^{G}$ corresponds to the "Gangster" semidefinite relaxation denoted by $\left(Q A P_{R 3}\right)$ in (Karisch 1995), and also used but denoted $\left(Q A P_{R 2}\right)$ in (Zhao et al. 1998, Rendl and Sotirov 2007). It contains $O\left(n^{3}\right)$ linear constraints. Note that one can also consider the equivalent semidefinite relaxation $\left(Q A P_{\mathcal{C}}\right)^{G}$ :

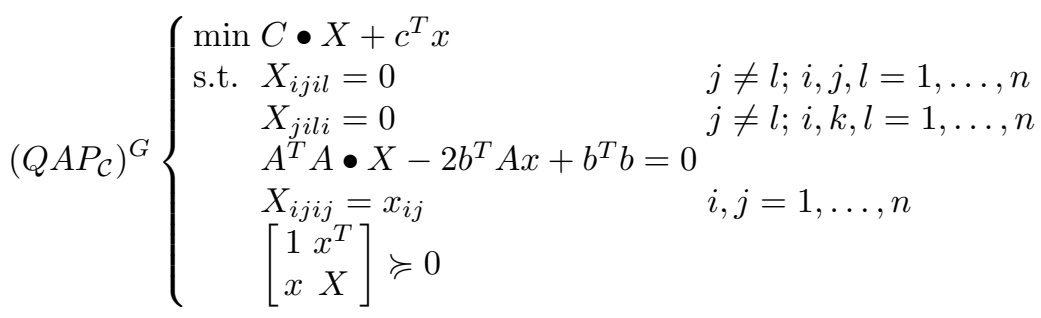

This semidefinite relaxation is stronger than $\left(Q A P_{\mathcal{P}}\right)^{O}$ and $\left(Q A P_{\mathcal{C}}\right)^{O}$, since the constraints in $\mathcal{G}$ obviously imply the constraints in $\mathcal{O}$.

For $\mathcal{K}=\mathcal{L S}=\left\{x_{i j} x_{k l} \geq 0: i, j, k, l \in\{1, \ldots, n\}\right\}$, we obtain the equivalent semidefinite relaxations $\left(Q A P_{\mathcal{C}}\right)^{L S}$ and $\left(Q A P_{\mathcal{P}}\right)^{L S}$ which involve $O\left(n^{4}\right)$ linear constraints. $\left(Q A P_{\mathcal{C}}\right)^{L S}$, which is thus $\left(Q A P_{\mathcal{C}}\right)^{G}$ with the additional constraints $X_{i j k l} \geq 0$ (for all $i j, k, l$ ), is used in a recent paper (de Klerk and Sotirov 2007), where the authors write the "Gangster" constraints as a single constraint thanks to these non-negativity constraints. Moreover, in this particular case, the constraints $X_{i j i j}=x_{i j}$ for all $i$ and $j$ are redundant and $A^{T} A \bullet X-2 b^{T} A x+b^{T} b=0$ simplifies to $\sum_{i, j} X_{i j i j}-2 \sum_{i, j} x_{i j}+n=0$.

Numerical results for $\left(Q A P_{\mathcal{P}}\right)^{L S}$ are given in (Roupin 2004) using the SDP solver SB (Helmberg 2004) based on the Spectral Bundle method (Helmberg and Rendl 2000). Note that the semidefinite relaxation proposed in this paper contains two more sets of constraints: $\sum_{i=1}^{n} \sum_{k=1}^{n} X_{i j k j}=1$ for $j$ in $\{1, \ldots, n\}$ 
and $\sum_{j=1}^{n} \sum_{k=1}^{n} X_{i j i k}=1$ for $i$ in $\{1, \ldots, n\}$. These constraints are redundant because they are implied by $(P 1)$ and $(P 2)$ and the assignment constraints. In fact, they are added to $\left(Q A P_{\mathcal{P}}\right)^{L S}$ because they speed up the solving process of the Spectral Bundle algorithm.

$$
\left(Q A P_{\mathcal{P}}\right)^{L S}\left\{\begin{array}{rll}
\min & C \bullet X+c^{T} x & \\
\text { s.t. } & \sum_{i=1}^{n} x_{i j}=1 & j=1, \ldots, n \\
& \sum_{j=1}^{n} x_{i j}=1 & i=1, \ldots, n \\
\left(P_{1}\right) & \sum_{i=1}^{n} X_{i j k l}=x_{k l} & \forall j, k, l=1, \ldots, n \\
\left(P_{2}\right) & \sum_{j=1}^{n} X_{i j k l}=x_{k l} & \forall i, k, l=1, \ldots, n \\
& X_{i j k l} \geq 0 & \forall i, j, k, l=1, \ldots, n \\
& X_{i j i j}=x_{i j} & i, j=1, \ldots, n \\
& {\left[\begin{array}{ll}
1 & x^{T} \\
x & X
\end{array}\right] \succeq 0} &
\end{array}\right.
$$

The relaxation $\left(Q A P_{\mathcal{P}}\right)^{L S}$ is also considered in (Burer and Vandenbussche 2006) where an efficient solving algorithm (based on an augmented Lagrangian approach) is used. One obtains an equivalent relaxation $\left(Q A P_{R 3}\right)$ (Karisch 1995, Zhao et al. 1998, Rendl and Sotirov 2007) by starting from $\left(Q A P_{R 1}\right)$ then by using the sets $\mathcal{G}$ and $\mathcal{L} \mathcal{S}$. In particular, it is easy to verify that the "Gangster" constraints are useless in $\left(Q A P_{\mathcal{P}}\right)^{L S}$. Indeed, when $l=j$ in constraint $(P 1)$ and $i=k$ in constraint $(P 2)$, since $X_{i j i j}=x_{i j}$ for all $i$ and $j$, one gets $\sum_{i=1 \mid i \neq k}^{n} X_{i j k j}=0$ for all $k$ and $j$, and $\sum_{j=1 \mid j \neq l}^{n} X_{i j i l}=0$ for all $i$ and $l$. All the $X_{i j k l}$ being non-negative, the "Gangster" constraints are satisfied. A direct proof of the equivalence between $\left(Q A P_{\mathcal{P}}\right)^{L S}$ and $\left(Q A P_{R 3}\right)$ is given in (Povh and Rendl 2006) without considering the equivalent Lagrangian relaxation $(D P)_{Q A P}^{\mathcal{L S}}$.

Now, one may think to add to $\left(Q A P_{\mathcal{P}}\right)^{L S}$ other standard linearization inequalities in order to obtain stronger semidefinite relaxations of the QAP (Rendl and Sotirov 2007). More precisely, one may consider $X_{i j k l} \leq x_{k l}$, and $x_{i j}+x_{k l}-$ $1 \leq X_{i j k l}$ for all $i, j, k, l$ in $\{1, \ldots, n\}$. First, the constraints $(P 1),(P 2)$, and the non-negativity constraints in $\left(Q A P_{\mathcal{P}}\right)^{L S}$ imply $X_{i j k l} \leq x_{k l}$. The second set of constraints is also satisfied by any feasible solution $(X, \bar{x})$ of $\left(Q A P_{\mathcal{P}}\right)^{L S}$, because one has $1-x_{i j}-x_{k l}+X_{i j k l}=\sum_{r \neq j} x_{i r}-x_{k l}+X_{i j k l}=\sum_{r \neq j} x_{i r}-\sum_{r \neq j} X_{i r k l}-$ $X_{i j k l}+X_{i j k l}=\sum_{r \neq j}\left(x_{i r}-X_{i r k l}\right) \geq 0$ (following a similar proof given in (Billionnet and Elloumi 1992)). As noticed in (de Klerk and Sotirov 2007), other triangle inequalities are also satisfied by the feasible solutions of these equivalent semidefinite programs. Consequently, if one wants to get stronger semidefinite relaxations other valid inequalities must be considered. In (Faye and Roupin 2005), two other sets $I_{C}^{\prime}$ and $I_{L}^{\prime}$ of cuts are used with $\left(Q A P_{\mathcal{P}}\right)^{L S}$ in a Branch-and-Cut algorithm for the QAP. Initially, these cuts were proposed in linear programming approach (Blanchard et al. 2003). The first set is:

$$
I_{C}^{\prime}: \quad \sum_{c \in C} X_{i j l c} \leq \sum_{k \in A} X_{i j h k}+\sum_{c \in C} \sum_{b \in B, b \neq c} X_{l c h b}
$$

where $i, h, l$ are distinct fixed row indices of $x, j$ is a column indice of $x$, $(A, B,\{j\})$ is a partition of the index set $\{1, \ldots, n\}$, and $C \subset B$. The second set is: 


$$
I_{L}^{\prime}: \quad \sum_{c \in L} X_{i j c l} \leq \sum_{h \in A} X_{i j h k}+\sum_{c \in L} \sum_{b \in B, b \neq c} X_{c l b k}
$$

where $j, k, l$ are distinct fixed column indices of $x, i$ is a row indice of $x$, $(A, B,\{i\})$ is a partition of the index set $\{1, \ldots, n\}$, and $L \subset B$. Thanks to the strong tailing off effect of SB (Helmberg 2004), the semidefinite programming solver used in (Faye and Roupin 2005), an approximate solution is obtained in a short time. Then, this solution is used to generate efficient cutting planes which obviously improve the bound, but also speed up the convergence of SB. Hence, for several instances the total computing time of the Branch-and-Cut algorithm is less than the computing time needed to solve $\left(Q A P_{\mathcal{P}}\right)^{L S}$ within the same accuracy (see Figure 4 in Section 5, and (Faye and Roupin 2005) for detailed computational results).

\section{Numerical issues}

In Section 4, for each different set $\mathcal{K}$, we have seen that one can obtain several sets of equivalent semidefinite relaxations for the QAP. Using this approach, we were able to survey and compare the semidefinite relaxations proposed for the QAP in a simple way. Nevertheless, as recalled in section 1, these relaxations have a different numerical behavior depending on the SDP solver. For all the semidefinite relaxations described in section 4 , computational results can be found in the corresponding references. Therefore here, we only illustrate this point in Figures 1, 2 and 3 for three classical instances of the QAP (Burkard, Karisch and Rendl 1997).
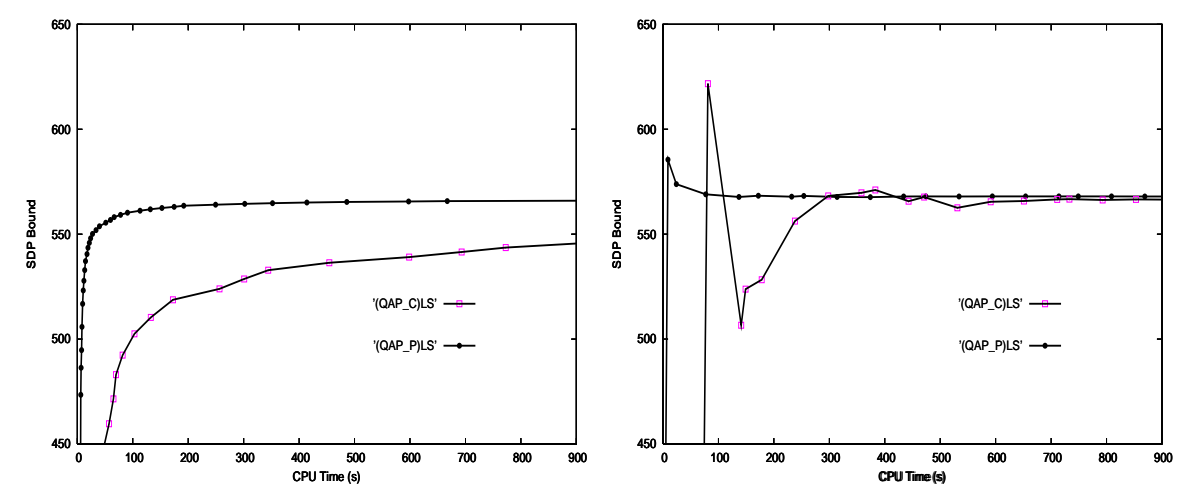

Fig. 1. Nug12 problem: bounds in dependence of CPU time when solving $\left(Q A P_{\mathcal{P}}\right)^{L S}$ and $\left(Q A P_{\mathcal{C}}\right)^{L S}$ with the Spectral Bundle algorithm SB (left side), and SDPLR (right side).

The semidefinite relaxations are defined in Section 4, and the numerical experiments have been carried out on a Pentium IV $2.2 \mathrm{MHz}$ computer with 1 
GoBytes of RAM under Linux. We have used three different solvers which are publically available : SB (Helmberg 2004), SDPLR (Burer and Monteiro 2003), and CSDP (Borchers 1999) (an interior-point method). For a given figure, all the semidefinite relaxations have the same optimal value.

As noticed in (Povh and Rendl 2006), if one wants to use an interior point method, then it is generally difficult to employ a semidefinite relaxation of the QAP with more than $O\left(n^{2}\right)$ constraints in an exact algorithm (for instance in a branch and bound setting). In this context, an alternative is to develop specific approaches, for instance by using representation theory to reduce in size the SDP relaxations of particular instances (de Klerk and Sotirov 2007). Other computational experiments (Faye and Roupin 2005, Burer and Vandenbussche 2006) suggest to choose a SDP solving method that can handle a large number of constraints (like the Spectral Bundle method or an augmented Lagrangian algorithm) and does not request the existence of interior points in both feasible regions of the primal and dual programs. By this way, it is possible to use in practice strong semidefinite relaxations for the QAP, especially the ones with $O\left(n^{4}\right)$ constraints. This point is illustrated in Figure 4 with numerical data from (Faye and Roupin 2005). In this particular problem, although the cutting planes do not improve the bound (which is in fact the optimal value), they actually speed up the solving process. This makes possible to use these semidefinite relaxations in an exact method (solving them approximately) by obtaining the bounds provided by weaker relaxations in a shorter time.

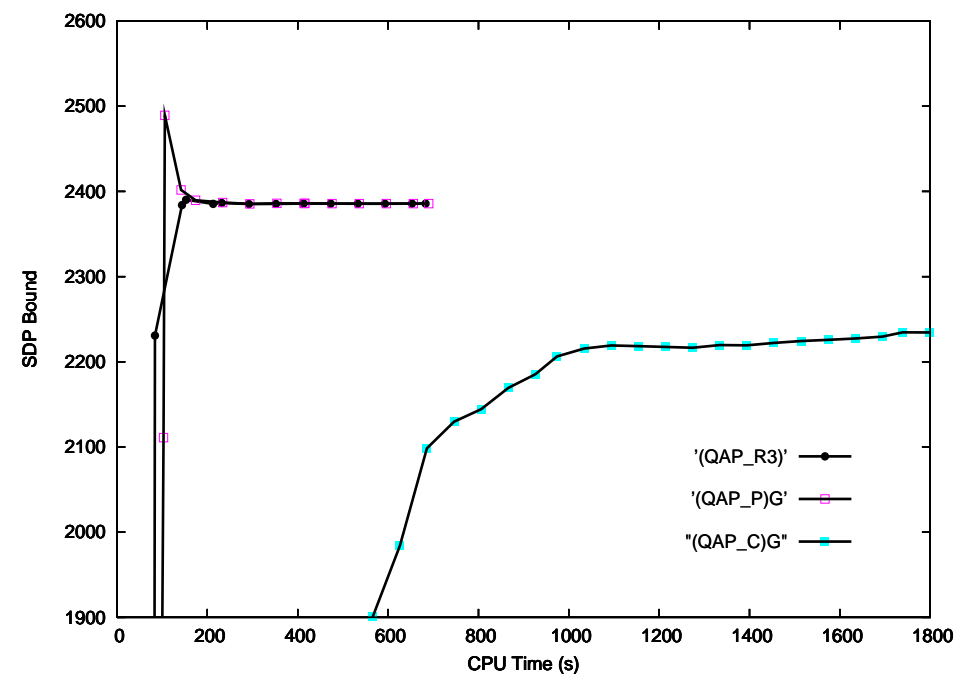

Fig. 2. Nug27 problem: bounds in dependence of CPU time when solving $\left(Q A P_{\mathcal{P}}\right)^{G}$, $\left(Q A P_{\mathcal{C}}\right)^{G}$ and $\left(Q A P_{R 3}\right)$ with SDPLR. 


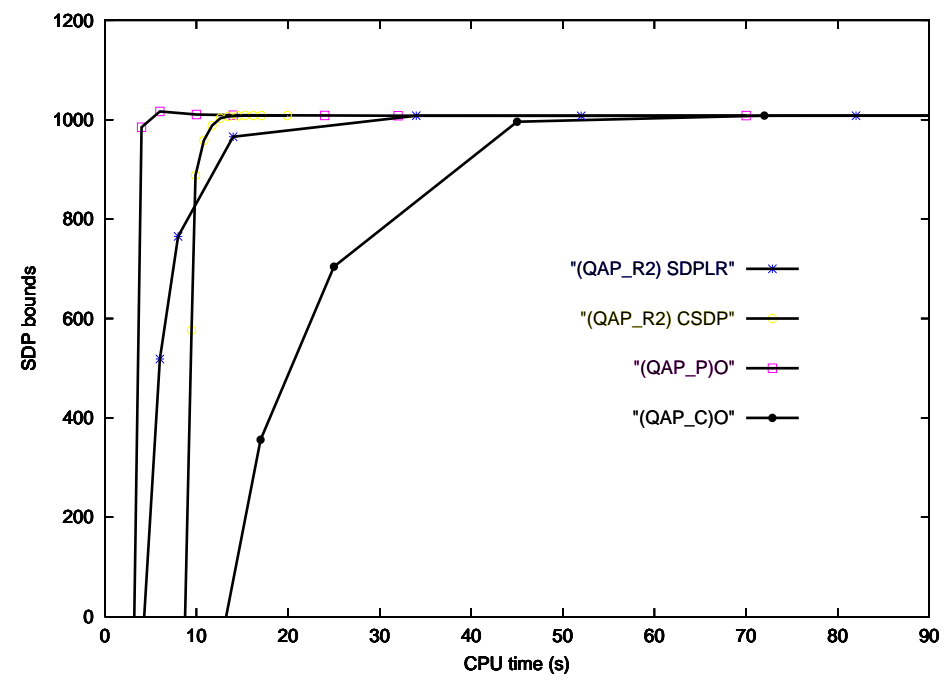

Fig. 3. Nug15 problem: bounds in dependence of CPU time when solving $\left(Q A P_{\mathcal{P}}\right)^{O}$, $\left(Q A P_{\mathcal{C}}\right)^{O}$ with SDPLR, and $\left(Q A P_{R 2}\right)$ with SDPLR and CSDP.

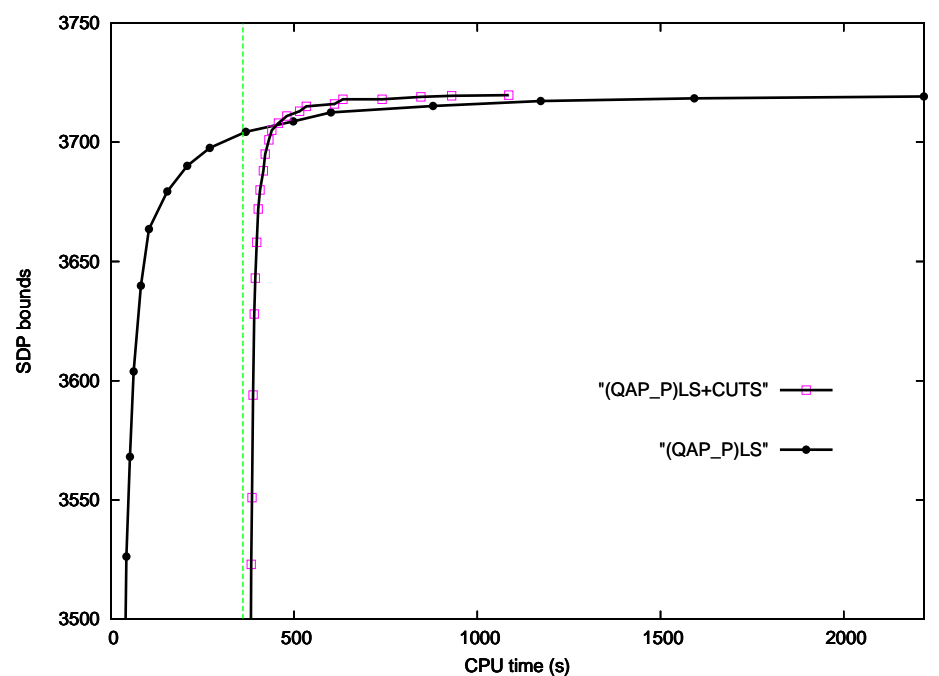

Fig. 4. Had16 problem: bounds in dependence of CPU time when solving $\left(Q A P_{\mathcal{P}}\right)^{L S}$ and $\left(Q A P_{\mathcal{P}}\right)^{L S}$ with a set of 262 cuts $I_{C}^{\prime}$ and $I_{L}^{\prime}$ with SB. CPU times of the second SDP are translated by $360 \mathrm{~s}$ (see the vertical dotted line) which corresponds to the time spent to solve approximately $\left(Q A P_{\mathcal{P}}\right)^{L S}$ and to generate the cuts. 


\section{Concluding remarks}

In this paper, taking advantage of a Lagrangian framework, we have been able to survey and compare semidefinite relaxations of the Quadratic Assignment Problem in a simple way. Moreover, we have illustrated the interest of considering partial Lagrangian relaxations to design various and strong semidefinite relaxations easily. This approach is very general and can be applied to many other combinatorial problems. With the arising of more and more efficient algorithms to solve SDP problems with many constraints, (see for instance the augmented Lagrangian approach proposed in (Malick, Povh, Rendl and Wiegele 2007) and improved in (Zhao, Sun, and Toh 2008)) it makes no doubt that semidefinite programming is one of the most promising approaches to solve exactly large instances of the Quadratic Assignment Problem.

\section{References}

Adams, W. P., Guignard, M., Hahn, P. M. and Hightower, W. L.: 2007, A level-2 reformulation-linearization technique bound for the quadratic assignment problem, European Journal of Operational Research 180(3), 983-996.

Adams, W. P. and Johnson, T. A.: 1994, Improved linear programming-based lower bounds for the quadratic assignment problem, Proceedings of the DIMACS Workshop on Quadratic Assignment Problems, Vol. 16, American Mathematical Society, pp. $43-75$.

Adams, W. P. and Sherali, H. D.: 1986, A tight linearization and an algorithm for zeroone quadratic progamming problems, Management Science 32(10), 1274-1290.

Anstreicher, K. M.: 2003, Recent advances in the solution of quadratic assignment problems, Mathematical Programming, Series B 97, 27-42.

Anstreicher, K. M. and Brixius, N.: 2001, A new bound for the quadratic assignment problem based on convex quadratic programming, Mathematical Programming 89, 341-357.

Anstreicher, K. M., Brixius, N., Goux, J.-P. and Linderoth, J.: 2002, Solving large quadratic assignment problems on computational grids, Mathematical Programming B 91, 563-588.

Beltran, C., Tadonki, C. and Vial, J. P.: 2006, Solving the p-median problem with a semi-lagrangian relaxation, Comput. Optim. Appl. 35(2), 239-260.

Billionnet, A. and Elloumi, S.: 1992, Best reduction of the quadratic semi-assignment problem, Discrete Applied Mathematics 109(3), 197-213.

Blanchard, A., Elloumi, S., Faye, A. and Wicker, N.: 2003, Un algorithme de génération de coupes pour le problème de l'affectation quadratique, INFOR 41(1), 35-49.

Borchers, B.: 1999, Csdp, a c library for semidefinite programming, Optimization Methods and Software 11(1), 613-623. Software available at https://projects.coinor.org/Csdp/.

Burer, S. and Monteiro, R. D. C.: 2003, A nonlinear programming algorithm for solving semidefinite programs via low-rank factorization, Mathematical Programming (series B) 95(2), 329-357. Software available at http://dollar.biz.uiowa.edu/ ${ }^{\sim}$ burer/software/SDPLR/. 
Burer, S. and Vandenbussche, D.: 2006, Solving lift-and-project relaxations of binary integer programs, SIAM Journal on Optimization 16(3), 726-750.

Burkard, R. E., Karisch, S. E. and Rendl, F.: 1997, Qaplib. a quadratic assignment problem library, J. of Global Opt. 10, 391-403. http://www.seas.upenn.edu/qaplib/.

Burkard, R. E. and Offermann, J.: 1977, Entwurf von schreibmaschinentastaturen mittels quadratischer zuordnungsprobleme, Mathematical Methods of Operations Research 21(4), B121-B132.

de Klerk, E. and Sotirov, R.: 2007, Exploiting group symmetry in semidefinite programming relaxations of the quadratic assignment problem, Discussion Paper 2007-44, Tilburg University, Center for Economic Research. Available at http://ideas.repec.org/p/dgr/kubcen/200744.html.

Eiselt, H. A. and Laporte, G.: 1991, Combinatorial optimization problem arising in dartboard design, The Journal of the Operational Research Society 42, 113-118.

Faye, A. and Roupin, F.: 2005, A cutting planes algorithm based upon a semidefinite relaxation for the quadratic assignment problem, Lecture Notes in Computer Science 3669, ESA 2005, 3-6 October, Majorca, Spain, pp. 850-861.

Faye, A. and Roupin, F.: 2007, Partial lagrangian for general quadratic programming, 4'OR, A Quarterly Journal of Operations Research 5(1), 75-88.

F.Çela: 1998, The Quadratic Assignment Problem: Theory and Algorithms, Kluwer, Massachessets, USA.

Geoffrion, A. M.: 1974, Lagrangean relaxation for integer programming, Mathematical Programming Study 2, 82-114.

Guignard, M.: 2003, Lagrangian relaxation, TOP 11(2), 151-228.

Helmberg, C.: 2004, A C++ implementation of the Spectral Bundle Method, http://www-user.tu-chemnitz.de/ ${ }^{\sim}$ helmberg/SBmethod/. Version 1.1.3.

Helmberg, C. and Rendl, F.: 2000, A spectral bundle method for semidefinite programming, SIAM J. Optim. 10(3), 673-696.

Kaibel, V.: 2000, Polyhedral methods for the quadratic assignment problem, in P. M. Pardalos and L. Pitsoulis (eds), Nonlinear Assignment Problems, Kluwer, pp. 109142.

Karisch, S.: 1995, Nonlinear approaches for the quadratic assignment and graph partition problems, Phd thesis, Graz University of Technology, Graz, Austria.

Koopmans, T. C. and Beckman, M.: 1957, Assignment problems and the location of economic activities, Econometric 25, 53-76.

Laurent, M.: 2003, A comparison of the sherali-adams, lovász-schrijver and lasserre relaxations for 0-1 programming, Mathematics of Operations Research 28, 470496.

Lemaréchal, C.: 2003, The omnipresence of lagrange, 4'OR, A Quarterly Journal of Operations Research 1, 7-25. Updated version in Annals in Operations Research 153(1):9-27, 2007.

Lemaréchal, C. and Oustry, F.: 2001, Semidefinite relaxations in combinatorial optimization from a lagrangian point of view. In Advances in Convex Analysis and Global Optimization, N. Hadjisavvas et P.M. Pardalos, Kluwer.

Litvinchev, I. S.: 2007, Refinement of lagrangian bounds in optimization problems, Computational Mathematics and Mathematical Physics 47(7), 1101-1107.

Loiola, E. M., de Abreu, N. M. M., Boaventura-Netto, P. O., Hahn, P. and Querido, T.: 2007, A survey for the quadratic assignment problem, EJOR 176(2), 657-690.

Malick, J., Povh, J., Rendl, F. and Wiegele, A.: 2007, Regularization methods for semidefinite programming, Submitted. Available on optimization-online, preprint 1800 . 
Poljak, S., Rendl, F. and Wolkowicz, H.: 1995, A recipe for semidefinite relaxation for (0,1)-quadratic programming, Journal of Global Optimization 7, 51-73.

Povh, J. and Rendl, F.: 2006, Copositive and semidefinite relaxations of the quadratic assignment problem, Online Paper available at www.optimization-online.org 1502, University Of Klagenfurt, Universitaetsstrasse 65-67, Austria.

Rendl, F. and Sotirov, R.: 2007, Bounds for the quadratic assignment problem using the bundle method, Mathematical Programming 109(2-3), 505-524.

Roupin, F.: 2004, From linear to semidefinite programming: an algorithm to obtain semidefinite relaxations for bivalent quadratic problems, Journal of Combinatorial Optimization 8(4), 469-493.

Steinberg, L.: 1961, The backboard wiring problem: A placement algorithm, SIAM Review 3, 37-50.

Zhao, Q., Karish, S. E., Rendl, F. and Wolkowicz, H.: 1998, Semidefinite programming relaxations for the quadratic assignment problem, Journal of Combinatorial Optimization 2(1), 71-109.

Zhao, X., Sun, D., and Toh, K.: 2008, A newton-cg augmented lagrangian method for semidefinite programming, preprint, National University of Singapore. 